\title{
Regularity 3 in edge ideals associated to bipartite graphs
}

\author{
Oscar Fernández-Ramos • Philippe Gimenez
}

Received: 26 February 2013 / Accepted: 15 August 2013 / Published online: 10 September 2013

(C) Springer Science+Business Media New York 2013

\begin{abstract}
We focus in this paper on edge ideals associated to bipartite graphs and give a combinatorial characterization of those having regularity 3 . When the regularity is strictly bigger than 3, we determine the first step $i$ in the minimal graded free resolution where there exists a minimal generator of degree $>i+3$, show that this step the highest degree of a minimal generator is $i+4$, and determine the corresponding graded Betti number $\beta_{i, i+4}$ in terms of the combinatorics of the graph. The results are then extended to the non-square-free case through polarization. We also study a family of ideals of regularity 4 that play an important role in our main result and whose graded Betti numbers can be completely described through closed combinatorial formulas.
\end{abstract}

Keywords Edge ideal · Bipartite graph · Castelnuovo-Mumford regularity · Graded Betti numbers · Independence complex · Stanley-Reisner ideal

\section{Introduction}

Studying homological invariants of monomial ideals in a polynomial ring $R=$ $\mathbb{K}\left[x_{1}, \ldots, x_{n}\right]$ by looking for combinatorial properties in discrete objects (graphs, hypergraphs, simplicial complexes, ...) associated to them is a well known technique that has been fruitfully exploited in the last decades (see for example [21] and the surveys $[13,17]$ and their references).

O. Fernández-Ramos

Dipartimento di Matematica, Università di Genova, Via Dodecaneso 35, 16146 Genova, Italy

e-mail: fernandez@dima.unige.it

P. Gimenez (ه)

Departamento de Álgebra, Análisis Matemático, Geometría y Topología, Facultad de Ciencias, Universidad de Valladolid, 47011 Valladolid, Spain

e-mail: pgimenez@agt.uva.es 
Classical objects used to relate combinatorics with monomial ideals are StanleyReisner ideals, simplicial or cellular resolutions and facet ideals. A monomial ideal generated by quadrics can be viewed as the facet ideal of a graph. When the graph is simple, i.e., has no loops, these ideals are called edge ideals and were first introduced in [20].

The homological invariants of a monomial ideal $I$ that we are interested in are those encoded in the minimal graded free resolution of the ideal, namely, the graded Betti numbers and the Castelnuovo-Mumford regularity. Considering the standard $\mathbb{N}$-grading on the polynomial ring $R$, the graded Betti number $\beta_{i, j}(I)$ is the number of minimal generators of degree $j$ in the $i$ th syzygy module of the ideal $I$. Denote by $u_{i}$ (resp. $l_{i}$ ) the maximal (resp. minimal) degree of a minimal generator in the $i$ th syzygy module. The fact that the resolution is minimal implies that $l_{i} \geq l_{0}+i$. The regularity of the ideal is $\operatorname{reg}(I):=\max \left\{u_{i}-i\right\}$. An interesting situation is when all minimal generators of $I$ have the same degree $l_{0}$ and $u_{i}=l_{i}=l_{0}+i$ for all $i$. In this case, $\operatorname{reg}(I)=l_{0}$ and we say that $I$ has an $l_{0}$-linear resolution.

There is a nice combinatorial characterization of edge ideals having 2-linear resolutions, i.e., having regularity 2 , in terms of the complement of the associated graph due to Fröberg ([8]). This result was recovered in [6] where, moreover, the least $i$ such that $u_{i}>i+2$ was characterized in a combinatorial way when the edge ideal does not have a linear resolution. The same characterization of this minimal $i$ was obtained independently in [7] where it was also shown that $u_{i}=i+3$ and where $\beta_{i, i+3}(I)$ was determined in terms of the complement of the graph. These results are recalled in the Sect. 2 (Theorem 2.3) together with all the required definitions and notations. We will also show in the same section that the graded Betti numbers of an arbitrary edge ideal $I$ satisfy the following property (Theorem 2.1): for every $i \geq 0$ and $j \geq i+2$,

$$
\beta_{i, j}(I)=\beta_{i, j+1}(I)=0 \Rightarrow \beta_{i+1, j+2}(I)=0 .
$$

It implies in particular that $u_{i+1} \leq u_{i}+2$ for all $i \geq 0$ (Corollary 2.1), a refinement of [7, Theorem 5.2]. This result has recently been generalized by Herzog and Srinivasan in [11].

The aim of this paper is to characterize edge ideals $I(G)$ associated to bipartite graphs having regularity 3 and determine, for those of regularity $>3$, the first step $i$ in the minimal resolution such that $u_{i}>i+3$. This will be done in Sect. 4 where we will also prove that, for this value of $i, u_{i}=i+4$ and $\beta_{i, i+4}(I(G))$ is the number of induced subgraphs of the bipartite complement of $G$ that are isomorphic to cycles of minimal length. The fundamental role played by these subgraphs is the reason why we previously devote Sect. 3 to study the minimal resolution of the edge ideal associated to the bipartite complement of an even cycle. We show that such an edge ideal has regularity 4 and give closed combinatorial formulas for all its graded Betti numbers. In particular, these results give a partial answer to [19, Question 3.5] on the regularity of bipartite edge ideals which are neither unmixed nor sequentially Cohen-Macaulay and provide a family of bipartite edge ideals whose regularity is strictly greater than the induced matching number plus one (Remark 3.2). In the last section, the previous results are extended to the non-square-free case. 
The dependence of the Betti numbers of edge ideals on the characteristic of the field $\mathbb{K}([15])$, even in the case of edge ideals associated to bipartite graphs ([4]), prevents the possibility of obtaining similar results for higher regularity.

\section{Preliminaries}

\subsection{Graphs and simplicial complexes}

We recall the relevant results and definitions about graphs and simplicial complexes required for this paper (see [5] and [18] for more details).

Consider a finite simple graph $G$ and denote by $E(G)$ and $V(G)$ its edge and vertex sets, respectively. We say that a subgraph $H$ of $G$ is induced on a subset $V^{\prime}$ of $V(G)$ if $V(H)=V^{\prime}$ and $E(H)=\left\{\{u, v\} \in E(G): u, v \in V^{\prime}\right\}$. We write $H=G\left[V^{\prime}\right]$, or $H<G$ when $H$ is an induced subgraph on an unspecified subset of vertices of $G$. The complement of $G$ is the graph $G^{c}$ with $V\left(G^{c}\right)=V(G)$ and $E\left(G^{c}\right)=\{\{u, v\}$ : $u, v \in V(G),\{u, v\} \notin E(G)\}$. Given a vertex $u \in V(G)$, we denote by $N_{G}(u)$ the set of vertices of $G$ adjacent to $u$ and, for a subset $W \subset V(G), N_{G}(W):=\bigcup_{u \in W} N_{G}(u)$. The degree of $u$, denoted by $\operatorname{deg}(u)$, is the number of elements in $N_{G}(u)$.

A connected graph $G$ whose vertices are all of degree two is called a $t$-cycle and denoted by $C_{t}$ where $t:=|V(G)|$ is referred to as the length of the cycle. A graph whose vertices have all degree one has necessarily $2 s$ vertices for some $s \geq 1$ and consists of $s$ disconnected edges. We denote it by $s K_{2}$.

Consider now a simplicial complex $\Delta$. Given a subset $W$ of its vertex set $V(\Delta)$, the induced subcomplex of $\Delta$ on $W$ is $\Delta[W]:=\{\sigma \in \Delta: \sigma \subset W\}$. If one has two subcomplexes $\Delta_{1}$ and $\Delta_{2}$ of $\Delta$ such that $\Delta=\Delta_{1} \cup \Delta_{2}$, there is a long exact sequence of reduced homologies, called the Mayer-Vietoris sequence,

$$
\cdots \longrightarrow \tilde{H}_{i}\left(\Delta_{0}\right) \longrightarrow \tilde{H}_{i}\left(\Delta_{1}\right) \oplus \tilde{H}_{i}\left(\Delta_{2}\right) \longrightarrow \tilde{H}_{i}(\Delta) \longrightarrow \tilde{H}_{i-1}\left(\Delta_{0}\right) \longrightarrow \cdots
$$

whenever $\Delta_{0}:=\Delta_{1} \cap \Delta_{2} \neq \emptyset$. Note that we use the simplified notation $\tilde{H}_{i}(\Delta)$, omitting the dependence of the homology groups on the field $\mathbb{K}$.

Definition 2.1 Given a simplicial complex $\Delta$ and $u, v \notin V(\Delta)$, consider the following two simplicial complexes:

- $v * \Delta:=\Delta \cup\{\{v\} \cup \sigma: \sigma \in \Delta\}$, the cone on the base $\Delta$ with apex $v$;

- $\Sigma_{u}^{v} \Delta:=\Delta \cup\{\{u\} \cup \sigma: \sigma \in \Delta\} \cup\{\{v\} \cup \sigma: \sigma \in \Delta\}$, the suspension of $\Delta$ on the vertices $u$ and $v$.

Proposition 2.1 (see, e.g., [18, Theorems 8.2 and 25.4])

1. $\tilde{H}_{i}(v * \Delta)=0, \forall i \geq 0$.

2. $\tilde{H}_{0}\left(\Sigma_{u}^{v} \Delta\right)=0$ and $\tilde{H}_{i}\left(\Sigma_{u}^{v} \Delta\right) \simeq \tilde{H}_{i-1}(\Delta), \forall i \geq 1$.

A subset $S \subset V(G)$ is called independent if $e \not \subset S, \forall e \in E(G)$. Associated to a graph $G$, one has its independence complex $\Delta(G)$ whose faces are the independent sets of $G$. For any $W \subset V(G)$, one has $\Delta(G)[W]=\Delta(G[W])$. 
Remark 2.1 A flag complex is a simplicial complex $\Delta$ such that, for any $\sigma \subset V(\Delta)$, if every pair of elements in $\sigma$ is a face of $\Delta$ then $\sigma$ is also a face of $\Delta$. In particular, a flag complex containing all pairs of vertices is necessarily a simplex. The independence complex of a graph is always a flag complex.

Definition 2.2 Set $\Delta:=\Delta(G)$. Associated to any $u \in V:=V(G)$ one has three induced subcomplexes of $\Delta$, called deletion, link and star, respectively:

- $\operatorname{del}_{\Delta}(u):=\{\sigma \in \Delta: u \notin \sigma\}=\Delta[V \backslash\{u\}]$;

- $\operatorname{link}_{\Delta}(u):=\{\sigma \in \Delta: u \notin \sigma$ and $\sigma \cup\{u\} \in \Delta\}=\Delta\left[V \backslash\left(N_{G}(u) \cup\{u\}\right)\right]$;

- $\operatorname{star}_{\Delta}(u):=\{\sigma \in \Delta: \sigma \cup\{u\} \in \Delta\}=\Delta\left[V \backslash N_{G}(u)\right]$.

For any vertex $v \in V(G), \operatorname{star}_{\Delta}(v)$ is a cone with apex $v$ and hence it is acyclic by Proposition 2.1.1. Since $\operatorname{del}_{\Delta}(v) \cup \operatorname{star}_{\Delta}(v)=\Delta$ and $\operatorname{del}_{\Delta}(v) \cap \operatorname{star}_{\Delta}(v)=\operatorname{link}_{\Delta}(v)$, we can apply (1) whenever $\operatorname{link}_{\Delta}(v) \neq \emptyset$ and get

$$
\begin{aligned}
\cdots & \rightarrow \tilde{H}_{i}\left(\operatorname{link}_{\Delta}(v)\right) \longrightarrow \tilde{H}_{i}\left(\operatorname{del}_{\Delta}(v)\right) \longrightarrow \tilde{H}_{i}(\Delta) \longrightarrow \tilde{H}_{i-1}\left(\operatorname{link}_{\Delta}(v)\right) \rightarrow \cdots \\
& \rightarrow \tilde{H}_{0}\left(\operatorname{link}_{\Delta}(v)\right) \longrightarrow \tilde{H}_{0}\left(\operatorname{del}_{\Delta}(v)\right) \longrightarrow \tilde{H}_{0}(\Delta) \rightarrow 0 .
\end{aligned}
$$

Let us focus now on bipartite graphs. A graph $G$ is bipartite if its vertex set can be partitioned into two disjoint sets, $V(G)=X \sqcup Y$, in such a way that any edge of $G$ has one vertex in $X$ and the other in $Y$. Let $G$ be a bipartite graph with $V(G)=X \sqcup Y$. Denote variables in $X$ by $x_{1}, \ldots, x_{n}$ and variables in $Y$ by $y_{1}, \ldots, y_{m}$. The biadjacency matrix of $G, M(G)=\left(a_{i, j}\right) \in \mathcal{M}_{n \times m}(\{0,1\})$, is defined by $a_{i, j}=1$ if $\left\{x_{i}, y_{j}\right\} \in$ $E(G)$, and $a_{i, j}=0$ otherwise. The bipartite complement of $G$ is the bipartite graph $G^{b c}$ with $V\left(G^{b c}\right)=X \sqcup Y$ and $E\left(G^{b c}\right)=\{\{x, y\}: x \in X, y \in Y,\{x, y\} \notin E(G)\}$. One has $M\left(G^{b c}\right)=\mathbf{1}_{n \times m}-M(G)$ where $\mathbf{1}_{n \times m}$ is the $n \times m$ matrix whose entries are all 1. Note that the bipartition $V(G)=X \sqcup Y$ is not unique if $G$ is not connected. The notions of biadjacency matrix or bipartite complement depend on the bipartition. In Sect. 4, we will restrict ourselves to connected bipartite graphs.

The next lemma will be useful to handle the homology of the independence complex of a bipartite graph $G$.

Lemma 2.1 Let $G$ be a bipartite graph with $V(G)=\left\{x_{1}, \ldots, x_{n}\right\} \sqcup\left\{y_{1}, \ldots, y_{m}\right\}$, $M(G)=\left(a_{i, j}\right)$, and set $\Delta:=\Delta(G)$.

1. If $M(G)$ has a row or a column of zeros, then $\tilde{H}_{i}(\Delta)=0, \forall i \geq 0$.

2. If there exist $r$ and $c$ such that $a_{r, c}=1$ and the rest of entries on the row $r$ and the column c are zeros, then $\tilde{H}_{i}(\Delta) \simeq \tilde{H}_{i-1}\left(\Delta\left[V(G) \backslash\left\{x_{r}, y_{c}\right\}\right]\right), \forall i>0$.

3. If $M(G)$ has more than one row (resp. column) and if the entries on the row $r$ (resp. column c) are all 1, then

$$
\tilde{H}_{i}(\Delta) \simeq \tilde{H}_{i}\left(\Delta\left[V(G) \backslash\left\{x_{r}\right\}\right]\right)\left(\operatorname{resp} . \tilde{H}_{i}(\Delta) \simeq \tilde{H}_{i}\left(\Delta\left[V(G) \backslash\left\{y_{c}\right\}\right]\right)\right), \quad \forall i \geq 0 .
$$

4. If $M(G)$ has a row $r$ (resp. column $c$ ) with a unique zero entry, say $a_{r, c}=0$, and if there is another zero entry on the column c (resp. row $r$ ), then

$$
\tilde{H}_{i}(\Delta) \simeq \tilde{H}_{i}\left(\Delta\left[V(G) \backslash\left\{x_{r}\right\}\right]\right)\left(\operatorname{resp} . \tilde{H}_{i}(\Delta) \simeq \tilde{H}_{i}\left(\Delta\left[V(G) \backslash\left\{y_{c}\right\}\right]\right)\right), \quad \forall i \geq 0 .
$$


5. If $M(G)$ has two rows $r$ and $r^{\prime}$ (resp. two columns $c$ and $c^{\prime}$ ) such that $\{j$ : $\left.a_{r, j}=0\right\} \subset\left\{j: a_{r^{\prime}, j}=0\right\}$ (resp. $\left.\left\{i: a_{i, c}=0\right\} \subset\left\{j: a_{i, c^{\prime}}=0\right\}\right)$, then

$$
\tilde{H}_{i}(\Delta) \simeq \tilde{H}_{i}\left(\Delta\left[V(G) \backslash\left\{x_{r}\right\}\right]\right)\left(\operatorname{resp} . \tilde{H}_{i}(\Delta) \simeq \tilde{H}_{i}\left(\Delta\left[V(G) \backslash\left\{y_{c}\right\}\right]\right)\right), \quad \forall i \geq 0
$$

Proof 1: The vertex $z$ of $G$ corresponding to the row or column of $M(G)$ with zero entries is isolated in $G$. Hence $\Delta$ is a cone with apex $z$, so it is acyclic by Proposition 2.1.1.

2: By 1, one has $\tilde{H}_{i}\left(\operatorname{del}_{\Delta}\left(y_{c}\right)\right)=\tilde{H}_{i}\left(\operatorname{del}_{\Delta}\left(x_{r}\right)\right)=0$. Since $\Delta=\operatorname{del}_{\Delta}\left(x_{r}\right) \cup$ $\operatorname{del}_{\Delta}\left(y_{c}\right)$ and $\operatorname{del}_{\Delta}\left(x_{r}\right) \cap \operatorname{del}_{\Delta}\left(y_{c}\right)=\operatorname{del}_{\operatorname{del}_{\Delta}\left(x_{r}\right)}\left(y_{c}\right)$, the result follows from the Mayer-Vietoris sequence (2).

3 and 4 are particular cases of 5 . Case 5 follows by applying again the MayerVietoris sequence (2), observing that $\operatorname{link}_{\Delta}\left(x_{r}\right)$ is acyclic by 1 .

Example 2.1 Set $\Sigma_{m}:=\Delta\left(m K_{2}\right)$. Then,

$$
\operatorname{dim}_{\mathbb{K}}\left(\tilde{H}_{i}\left(\Sigma_{m}\right)\right)= \begin{cases}1 & \text { if } i=m-1 \\ 0 & \text { otherwise }\end{cases}
$$

This can be shown by induction on $m \geq 1$ as follows. Since $\Sigma_{1}$ consists of two disjoint vertices, $\tilde{H}_{0}\left(\Sigma_{1}\right) \simeq \mathbb{K}$ and $\tilde{H}_{i}\left(\Sigma_{1}\right)=0$ for all $i>0$. If $m>1, \tilde{H}_{0}\left(\Sigma_{m}\right)=0$ because $\Sigma_{m}$ is connected. For $i>0$, applying Lemma 2.1.2, one gets that $\tilde{H}_{i}\left(\Sigma_{m}\right) \simeq$ $\tilde{H}_{i-1}\left(\Sigma_{m-1}\right)$ and the result follows.

\subsection{Some properties of the graded Betti numbers of an edge ideal}

The (Castelnuovo-Mumford) regularity of $I$ is defined in terms of its graded Betti numbers as $\operatorname{reg}(I):=\max \left\{j-i: \beta_{i, j}(I) \neq 0\right\}$. The graded Betti numbers of $I$ are usually arranged in a table called the Betti diagram of $I$ where $\beta_{i, j}(I)$ is placed in the $i$ th column and $(j-i)$ th row of the table. The index of the last nonzero row in the Betti diagram of $I$ is its regularity.

If we provide $R$ with the usual $\mathbb{N}^{n}$-multigrading, any monomial ideal $I$ has a minimal multigraded free resolution. Its multigraded Betti numbers, $\beta_{i, \boldsymbol{m}}(I)$, are defined as the number of minimal generators of degree $\boldsymbol{m} \in \mathbb{N}^{n}$ in the $i$ th syzygy module.

There is a one-to-one correspondence between square-free monomial ideals generated in degree 2 and simple graphs. Associated to a simple graph $G$, one has the edge ideal $I(G)$ generated by the monomials of the form $x_{i} x_{j}$ with $\left\{x_{i}, x_{j}\right\} \in E(G)$. The ideal $I(G)$ is the Stanley-Reisner ideal of $\Delta(G)$, that is, $I(G)=I_{\Delta(G)}$. The multigraded Betti numbers of $I(G)$ can be expressed in terms of the reduced homology of $\Delta(G)$ using Hochster's Formula ([12, Theorem (5.1)]) that we recall now. For any $\boldsymbol{m} \in \mathbb{N}^{n}$ and $i \geq 0$, one has $\beta_{i, \boldsymbol{m}}(I(G))=0$ if the monomial $\boldsymbol{x}^{\boldsymbol{m}}:=x_{1}^{m_{1}} \cdots x_{n}^{m_{n}}$ is not square-free, and

$$
\beta_{i, \boldsymbol{m}}(I(G))=\operatorname{dim}_{\mathbb{K}} \tilde{H}_{|\boldsymbol{m}|-i-2}(\Delta(G)[W])
$$


otherwise, where $W:=\left\{x_{j} \in V(G): m_{j}=1\right\}$. The graded Betti numbers of $I(G)$ are then given by the following formula:

$$
\beta_{i, j}(I(G))=\sum_{W \subset V(G),|W|=j} \operatorname{dim}_{\mathbb{K}}\left(\tilde{H}_{j-i-2}(\Delta(G)[W])\right) .
$$

Hochster's Formula is a powerful tool when one wants to get information on the Betti numbers of edge ideals. For example, it can be used to prove the following property on the graded Betti numbers of an edge ideal $I(G)$ :

Theorem 2.1 For any $i \geq 0$ and any $j \geq i+2$, if $\beta_{i, j}(I(G))=\beta_{i, j+1}(I(G))=0$ then $\beta_{i+1, j+2}(I(G))=0$.

Proof Set $\Delta:=\Delta(G)$ and suppose that $\beta_{i+1, j+2}(I(G)) \neq 0$. By (4), there exists $W \subset V(G)$ with $|W|=j+2$ such that $\operatorname{dim}_{\mathbb{K}} \tilde{H}_{j-i-1}(\Delta[W])>0$ and hence $\Delta[W]$ is not a simplex. As $\Delta[W]=\Delta(G[W])$ is a flag complex, there exist $u, v \in W$ such that $\{u, v\} \notin \Delta(G)[W]$ by Remark 2.1. Consider then the following decomposition of $\Delta[W]$,

$$
\Delta[W]=\Delta[W \backslash\{u\}] \cup \Delta[W \backslash\{v\}],
$$

with $\Delta[W \backslash\{u\}] \cap \Delta[W \backslash\{v\}]=\Delta[W \backslash\{u, v\}]$ which is not empty since $|W|=$ $j+2>2$. Invoking Hochster's Formula (4) again, one has $\tilde{H}_{j-i-1}(\Delta[W \backslash\{u\}])=$ $\tilde{H}_{j-i-1}(\Delta[W \backslash\{v\}])=0$ because $\beta_{i, j+1}(I)=0$ and $\tilde{H}_{j-i-2}(\Delta[W \backslash\{u, v\}])=0$ because $\beta_{i, j}(I)=0$. Using the Mayer-Vietoris sequence (1), one gets that $\tilde{H}_{j-i-1}(\Delta[W])=0$, a contradiction.

Note that Theorem 2.1 can easily be extended to non-square-free monomial ideals generated in degree two through polarization (see Sect. 5). The following direct consequence answers a question by Aldo Conca who asked if [7, Theorem 5.2] could be improved in this direction. In [1], Avramov, Conca and Iyengar proved bounds for the syzygies of Koszul algebras and that question arose in this context.

Corollary 2.1 Let I be a monomial ideal generated in degree two and denote by $u_{i}$ the maximal degree of a minimal generator in its ith syzygy module. Then, for all $i \geq 0, u_{i+1} \leq u_{i}+2$.

When an edge ideal $I(G)$ has a linear resolution, all the nonzero entries in its Betti diagram are located on the first row. Fröberg proved that an edge ideal $I(G)$ has a linear resolution if and only if the graph $G^{c}$ is chordal. We can rephrase this nice combinatorial characterization as follows:

Theorem 2.2 [8] An edge ideal $I(G)$ has regularity 2 if and only if $G^{c}$ does not have induced cycles of length $\geq 4$.

In [6], the authors go one step further and show that if $\operatorname{reg}(I(G))>2$, the nonlinear syzygies appear for the first time at the $(t-3)$ th step of the resolution where 
$t$ is the minimal length of an induced cycle in $G^{c}$. This result is contained in the following stronger statement:

Theorem 2.3 [7] If $I(G)$ is an edge ideal with $\operatorname{reg}(I(G))>2$, let $t \geq 4$ be the minimal length of an induced cycle in $G^{c}$. Then:

- $\beta_{i, j}(I(G))=0$ for all $i<t-3$ and $j>i+2$;

- $\beta_{t-3, t}(I(G))=\mid\left\{\right.$ induced $t$-cycles in $\left.G^{c}\right\} \mid$;

- $\beta_{t-3, j}(I(G))=0$ for all $j>t$;

- for any $\boldsymbol{m} \in \mathbb{N}^{n}$ such that $|\boldsymbol{m}|=t$, one has $\beta_{t-3, \boldsymbol{m}}(I(G))=1$ if $\boldsymbol{m} \in\{0,1\}^{n}$ and $G^{c}[W] \simeq C_{t}$ where $W:=\left\{x_{i}: m_{i}=1\right\}$. Otherwise, $\beta_{t-3, m}(I(G))=0$.

\section{Bipartite complement of a cycle of even length}

Induced cycles in $G^{c}$ play an important role in Theorem 2.3. That is why we focused on the family of edge ideals associated to complements of cycles in [7, Proposition 3.1], where we gave closed combinatorial formulas for all its graded Betti numbers. Following the same philosophy, we focus now on graphs that are the bipartite complement of an even cycle because they will play a fundamental role in our main Theorem 4.2. The analogue of [7, Proposition 3.1] is Theorem 3.1 which is a direct consequence of Propositions 3.3, 3.4 and 3.5, which will be proved in this section.

Theorem 3.1 The edge ideal I associated to the bipartite complement of an even cycle of length $t:=2 s \geq 6$ has regularity 4 and its Betti diagram is

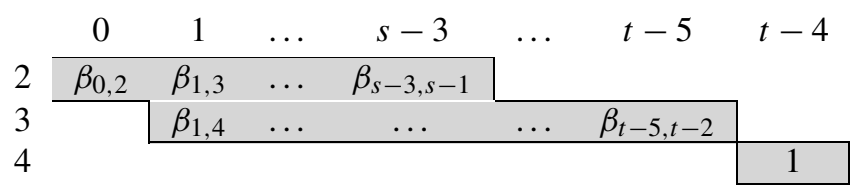

where the nonzero entries are located in the shadowed area. Moreover, $\beta_{j-2, j}$ for $2 \leq j \leq s-1$, and $\beta_{j-3, j}$ for $4 \leq j \leq t-2$, are given, respectively, by the following closed combinatorial formulas:

$$
\begin{aligned}
& \beta_{j-2, j}=\sum_{k=1}^{j-1} \sum_{c=1}^{k} \frac{s}{c}\left(\begin{array}{c}
k-1 \\
c-1
\end{array}\right)\left(\begin{array}{c}
s-k-1 \\
c-1
\end{array}\right)\left(\begin{array}{c}
s-k-c \\
j-k
\end{array}\right), \\
& \beta_{j-3, j}=\sum_{m=2}^{\lfloor j / 2\rfloor} t \frac{m-1}{m}\left(\begin{array}{c}
t-j-1 \\
m-1
\end{array}\right) \sum_{a=0}^{j-2 m}\left(\begin{array}{c}
j-m-a-1 \\
m-1
\end{array}\right)\left(\begin{array}{c}
t-j-m \\
a
\end{array}\right) .
\end{aligned}
$$

Let $G:=C_{2 s}^{b c}$ be the bipartite complement of an even cycle $C_{2 s}$ with $s \geq 3$. The vertices and edges of $C_{2 s}$ will be $V=X \sqcup Y$ with $X=\left\{x_{1}, \ldots, x_{s}\right\}, Y=\left\{y_{1}, \ldots, y_{s}\right\}$ and $\left\{\left\{x_{1}, y_{1}\right\},\left\{y_{1}, x_{2}\right\}, \ldots,\left\{y_{s}, x_{1}\right\}\right\}$, respectively. Every column and row of $M(G)$ 
has exactly two zero entries:

$$
M(G)=\left(\begin{array}{cccccc}
0 & 1 & \ldots & \ldots & 1 & 0 \\
0 & 0 & \ddots & & \vdots & 1 \\
1 & 0 & 0 & \ddots & \vdots & \vdots \\
\vdots & \ddots & \ddots & \ddots & 1 & \vdots \\
\vdots & & \ddots & 0 & 0 & 1 \\
1 & \ldots & \ldots & 1 & 0 & 0
\end{array}\right) .
$$

In order to use Hochster's Formula to determine the graded Betti numbers of $I(G)$, we need to compute the reduced simplicial homologies $\tilde{H}_{i}(\Delta(G[W]))$ for all subsets $W$ of $V$. This task will be achieved in Propositions 3.1 and 3.2 when $W=V$ and when $W$ is a proper subset, respectively. Their proofs require two technical lemmas.

Lemma 3.1 For every $v \in V, \operatorname{del}_{\Delta(G)}(v)$ is acyclic.

Proof Without loss of generality, let us choose $v=x_{1}$. As observed in Definition 2.2, $\operatorname{del}_{\Delta(G)}\left(x_{1}\right)$ is the independence complex of $G\left[V \backslash\left\{x_{1}\right\}\right]$ whose biadjacency matrix $N$ is obtained by removing the first row of $M(G)$. Observe that the first and last columns of $N$ satisfy the condition in Lemma 2.1.4 and hence can be removed. Again, the first and last rows of this new matrix satisfy the same condition and we remove them. Recursively, when $s$ is odd (respectively even), we reduce the computation of the homology to the case of the independence complex of a graph whose biadjacency matrix is a $2 \times 3$ (respectively, $3 \times 2$ ) matrix whose central column (respectively row) has its two entries equal to zero. But then $\operatorname{del}_{\Delta(G)}(v)$ is acyclic by Lemma 2.1.1.

Lemma 3.2 For all $m \geq 1$,

$$
\operatorname{dim}_{\mathbb{K}}\left(\tilde{H}_{i}\left(\Delta\left(\left(m K_{2}\right)^{b c}\right)\right)\right)= \begin{cases}m-1 & \text { if } i=1, \\ 0 & \text { otherwise }\end{cases}
$$

Proof The entries of the square matrix $M\left(\left(m K_{2}\right)^{b c}\right)$ are all 1 except those on the principal diagonal that are zero. Set $\Theta_{m}:=\Delta\left(\left(m K_{2}\right)^{b c}\right)$. Since $\Theta_{m}$ is connected, $\operatorname{dim}_{\mathbb{K}}\left(\tilde{H}_{0}\left(\Theta_{m}\right)\right)=0$ for all $m \geq 1$.

In order to determine the homology for $i \geq 1$, consider the family of subcomplexes of $\Theta_{m}, F=\left\{\Theta_{m}[X], \Theta_{m}[Y],\left\{x_{1}, y_{1}\right\}, \ldots,\left\{x_{m}, y_{m}\right\}\right\}$ whose elements we index by $x, y, z_{1}, \ldots, z_{m}$. Recall that the nerve of $F, N(F)$, is the simplicial complex on the vertex set $V_{F}:=\left\{x, y, z_{1}, \ldots, z_{m}\right\}$ whose faces are the subsets of $V_{F}$ such that the intersection of the corresponding elements in $F$ is non-empty. The simplicial complex $N(F)$ has $2 m$ facets, $\left\{x, z_{i}\right\}$ and $\left\{y, z_{i}\right\}$ for all $i=1, \ldots, m$. Since $\Theta_{m}=\bigcup_{i \in V_{F}} F_{i}$, applying the Nerve Theorem (see, for example, [2, Theorem 10.6]), one gets that $\tilde{H}_{i}\left(\Theta_{m}\right) \simeq \tilde{H}_{i}(N(F)), \forall i \geq 0$. On the other hand, $N(F)=\Sigma_{x}^{y}\left\langle\left\{z_{1}\right\}, \ldots,\left\{z_{m}\right\}\right\rangle$ and hence, by Proposition 2.1.2, $\tilde{H}_{i}(N(F)) \simeq \tilde{H}_{i-1}\left(\left\langle\left\{z_{1}\right\}, \ldots,\left\{z_{m}\right\}\right\rangle\right), \forall i \geq 1$, and the result follows. 


\section{Proposition 3.1}

$$
\operatorname{dim}_{\mathbb{K}}\left(\tilde{H}_{i}(\Delta(G))\right)= \begin{cases}1 & \text { if } i=2 \\ 0 & \text { otherwise }\end{cases}
$$

Proof Since $\Delta(G)$ is connected, $\operatorname{dim}_{\mathbb{K}}\left(\tilde{H}_{0}(\Delta(G))\right)=0$. For $i>0$, using the sequence (2), Lemma 3.1 implies that $\tilde{H}_{i}(\Delta(G)) \simeq \tilde{H}_{i-1}\left(\operatorname{link}_{\Delta(G)}(v)\right)$ for every vertex $v$ of $G$. The biadjacency matrix (or its transpose) of $G\left[V \backslash\left(N_{G}(v) \cup\{v\}\right)\right]$ is a $2 \times(s-1)$ matrix with exactly two zero entries, one in each row and located on two different columns. Applying Lemma 2.1.3 (if $s>3$ ) as many times as necessary, one gets that $\tilde{H}_{i}(\Delta(G)) \simeq \tilde{H}_{i-1}\left(\Sigma_{2}\right)$ and the result follows from Example 2.1.

Let $W$ be now a proper subset of $V=X \sqcup Y$. Set $W_{X}:=W \cap X, W_{Y}:=W \cap Y$, and denote by $k_{W}$ the number of connected components of $C_{2 s}[W]$ that are not isolated vertices. Note that if $k_{W} \neq 0$, then $W_{X} \neq \emptyset$ and $W_{Y} \neq \emptyset$.

\section{Proposition 3.2}

1. If $W_{X}=\emptyset$ or $W_{Y}=\emptyset$ then $\Delta(G[W])$ is acyclic.

2. If $W_{X} \neq \emptyset, W_{Y} \neq \emptyset$ and $k_{W}=0$ then

$$
\operatorname{dim}_{\mathbb{K}}\left(\tilde{H}_{i}(\Delta(G[W]))\right)= \begin{cases}1 & \text { if } i=0, \\ 0 & \text { otherwise } .\end{cases}
$$

3. If $k_{W}>0$ then

$$
\operatorname{dim}_{\mathbb{K}}\left(\tilde{H}_{i}(\Delta(G[W]))\right)= \begin{cases}k_{W}-1 & \text { if } i=1, \\ 0 & \text { otherwise }\end{cases}
$$

Remark 3.1 Observe that $G^{c}=C_{2 s} \cup K_{X} \cup K_{Y}$, where $K_{A}$ denotes the complete graph on a set of vertices $A \subset V$. Thus, $G^{c}[W]=C_{2 s}[W] \cup K_{W_{X}} \cup K_{W_{Y}}$. Since $K_{W_{X}}$ and $K_{W_{Y}}$ are connected, if one of them is empty or if they are connected to each other in $G^{c}[W]$, i.e., if $k_{W} \neq 0$, then $G^{c}[W]$ is connected. Otherwise, $K_{W_{X}}$ and $K_{W_{Y}}$ are its connected components. Thus, the condition in Proposition 3.2.2 is satisfied if and only if $G^{c}[W]$ is not connected. When $W_{X} \neq \emptyset$ and $W_{Y} \neq \emptyset$, denote by $M[W]:=M(G[W])$. It is easy to check that if $W_{X} \neq \emptyset$ and $W_{Y} \neq \emptyset$, then

$$
k_{W}=0 \quad \Leftrightarrow \quad N_{C_{2 s}}\left(W_{X}\right) \cap W_{Y}=\emptyset \quad \Leftrightarrow \quad M[W] \text { has no zero entries. }
$$

Recall that $N_{C_{2 s}}\left(W_{X}\right)$ is the set of neighbors of the elements of $W_{X}$ in $C_{2 s}$.

Proof (of Proposition 3.2) If $W_{X}=\emptyset$ or $W_{Y}=\emptyset, \Delta(G[W])$ is a simplex and 1 follows. Assume now that $W_{X} \neq \emptyset, W_{Y} \neq \emptyset$ and $k_{W}=0$. Then, $M[W]$ has no zero entries by Remark 3.1 and, by Lemma 2.1 .3 , for all $i \geq 0, \tilde{H}_{i}(\Delta(G[W])) \simeq$ $\tilde{H}_{i}\left(\Delta\left(K_{2}\right)\right)=\tilde{H}_{i}\left(\Sigma_{1}\right)$. So 2 follows from Example 2.1 .

Assume now that $M[W]$ has at least one zero entry. First observe that the number of zeros in any row and column of $M[W]$ is at most two (and that for at least two of the columns or rows, it is one). By Lemma 2.1.3, the dimension of the 
reduced homologies will not change if we remove from $M[W]$ any row and any column with no zero. In other words, since a row or a column of $M[W]$ with no zero entry corresponds to an isolated vertex in $C_{2 s}[W]$, if $W^{\prime}$ is the subset of $W$ formed by all the elements in $W$ that are not isolated vertices in $C_{2 s}[W]$, one has $\tilde{H}_{i}(\Delta(G[W])) \simeq \tilde{H}_{i}\left(\Delta\left(G\left[W^{\prime}\right]\right)\right)$. Moreover, $k_{W}=k_{W^{\prime}}$. Using now Lemma 2.1.4, one can remove from $M\left[W^{\prime}\right]$ any row (resp. column) with exactly one zero and such that in the column (resp. row) where this zero is located, there is another zero. Such a row or column of $M\left[W^{\prime}\right]$ corresponds to a vertex of $C_{2 s}\left[W^{\prime}\right]$ of degree one whose (unique) neighbor is of degree two. Removing such a vertex does not change the number of connected components of $C_{2 s}\left[W^{\prime}\right]$ and it creates in $C_{2 s}\left[W^{\prime}\right]$ a vertex of the same kind, until we reach a vertex of degree one whose neighbor also has degree one. Thus, $\tilde{H}_{i}(\Delta(G[W])) \simeq \tilde{H}_{i}\left(\Delta\left(\left(k_{W} K_{2}\right)^{b c}\right)\right)$ for all $i \geq 0$. The result is now a direct consequence of Lemma 3.2.

As a straightforward consequence, the ideal $I\left(C_{2 s}^{b c}\right)$ has regularity 4 and $\beta_{2 s-4,2 s} \times$ $\left(I\left(C_{2 s}^{b c}\right)\right)=1$ is the only nonzero entry on the last row of its Betti diagram.

\section{Proposition 3.3}

- $\beta_{i, j}\left(I\left(C_{2 s}^{b c}\right)\right)=0$ if $j>i+4$;

$$
\beta_{i, i+4}\left(I\left(C_{2 s}^{b c}\right)\right)= \begin{cases}1 & \text { if } i=2 s-4 \\ 0 & \text { otherwise }\end{cases}
$$

Proof Using Propositions 3.1 and 3.2, one gets that $\operatorname{dim}_{\mathbb{K}}\left(\tilde{H}_{i}(\Delta(G[W]))\right)=0$, for all $W \subset V$ and $i \notin\{0,1,2\}$. Moreover, $\operatorname{dim}_{\mathbb{K}}\left(\tilde{H}_{2}(\Delta(G[W]))\right) \neq 0$ if and only if $W=V$ and $\operatorname{dim}_{\mathbb{K}}\left(\tilde{H}_{2}(\Delta(G))\right)=1$. The result then follows from (4).

In order to complete the description of the Betti diagram of $I\left(C_{2 s}^{b c}\right)$, one has to determine the graded Betti numbers on the first two rows, i.e., $\beta_{i, j}\left(I\left(C_{2 s}^{b c}\right)\right)$ for $i+2 \leq$ $j \leq i+3$.

We start with the first row. Using Hochster's Formula (4) and Proposition 3.2.2, one needs to determine all the proper subsets $W$ of $V$ such that $W_{X} \neq \emptyset, W_{Y} \neq \emptyset$ and $G^{c}[W]$ is not connected. Indeed, $\beta_{i, i+2}(I(G))$ is the number of induced subgraphs $G^{c}[W]$ on $i+2$ vertices that are nonconnected.

Let us denote by $C_{X}$ the cycle on the vertex set $X$ whose edges are $\left\{x_{1}, x_{2}\right\}$, $\left\{x_{2}, x_{3}\right\}, \ldots,\left\{x_{s}, x_{1}\right\}$. Note that the edges of $C_{X}$ correspond to the pairs $\left\{x_{i}, x_{j}\right\}$ of elements in $X$ such that $N_{C_{2 s}}\left(x_{i}\right) \cap N_{C_{2 s}}\left(x_{j}\right) \neq \emptyset$.

Lemma 3.3 Assume that $G^{c}[W]$ is not connected.

1. There exists $x \in W_{X}$ such that $N_{C_{2 s}}(x) \not \subset N_{C_{2 s}}\left(W_{X} \backslash\{x\}\right)$.

2. $\left|N_{C_{2 s}}\left(W_{X}\right)\right|=\left|W_{X}\right|+\left|\operatorname{comp}\left(C_{X}\left[W_{X}\right]\right)\right|$ where $\operatorname{comp}\left(C_{X}\left[W_{X}\right]\right)$ is the set of connected components of $C_{X}\left[W_{X}\right]$.

Proof If $N_{C_{2 s}}(x) \subset N_{C_{2 s}}\left(W_{X} \backslash\{x\}\right)$ for some $x \in W_{X}$, then $N_{C_{X}}(x) \subset W_{X}$. Thus, if $N_{C_{2 s}}(x) \subset N_{C_{2 s}}\left(W_{X} \backslash\{x\}\right)$ for all $x \in W_{X}$, then $W_{X}=X$ and one cannot have $W_{Y} \neq \varnothing$ 
and $N_{C_{2 s}}\left(W_{X}\right) \cap W_{Y}=\emptyset$. This implies that $G^{c}[W]$ is connected by Remark 3.1 and 1 follows.

We prove 2 by induction on $r:=\left|W_{X}\right|$. If $W_{X}=\{x\}$ then $\left|N_{C_{2 s}}(x)\right|=2,|\{x\}|=1$, $\left|\operatorname{comp}\left(C_{X}[\{x\}]\right)\right|=1$ and the statement holds. Consider now $W$ such that $\left|W_{X}\right|=$ $r>1$ and assume that the statement holds for subsets $X^{\prime}$ such that $\left|W_{X^{\prime}}\right|=r-1$. By 1 , there exists $x_{0} \in W_{X}$ such that $N_{C_{2 s}}\left(x_{0}\right) \not \subset N_{C_{2 s}}\left(W_{X} \backslash\left\{x_{0}\right\}\right)$. There are two possibilities:

- If $N_{C_{2 s}}\left(x_{0}\right) \cap N_{C_{2 s}}\left(W_{X} \backslash\left\{x_{0}\right\}\right) \neq \emptyset$, i.e., if $x_{0}$ is connected in $C_{X}$ to some $x \in W_{X} \backslash\left\{x_{0}\right\}$, then $\left|N_{C_{2 s}}\left(W_{X}\right)\right|=\left|N_{C_{2 s}}\left(W_{X} \backslash\left\{x_{0}\right\}\right)\right|+1$. In this case, $\left|\operatorname{comp}\left(C_{X}\left[W_{X}\right]\right)\right|=\left|\operatorname{comp}\left(C_{X}\left[W_{X} \backslash\left\{x_{0}\right\}\right]\right)\right|$

- Otherwise, $\left|N_{C_{2 s}}\left(W_{X}\right)\right|=\left|N_{C_{2 s}}\left(W_{X} \backslash\left\{x_{0}\right\}\right)\right|+2$ and $\left|\operatorname{comp}\left(C_{X}\left[W_{X}\right]\right)\right|=$ $\left|\operatorname{comp}\left(C_{X}\left[W_{X} \backslash\left\{x_{0}\right\}\right]\right)\right|+1$.

In both cases, applying our inductive hypothesis, one gets that $\left|N_{C_{2 s}}\left(W_{X}\right)\right|=$ $\left|W_{X} \backslash\left\{x_{0}\right\}\right|+\left|\operatorname{comp}\left(C_{X}\left[W_{X}\right]\right)\right|+1=\left|W_{X}\right|+\left|\operatorname{comp}\left(C_{X}\left[W_{X}\right]\right)\right|$.

The first row of the Betti diagram is described in the following result.

\section{Proposition 3.4}

1. For all $j \geq s, \beta_{j-2, j}\left(I\left(C_{2 s}^{b c}\right)\right)=0$.

2. For $j=2, \ldots, s-1$,

$$
\beta_{j-2, j}\left(I\left(C_{2 s}^{b c}\right)\right)=\sum_{k=1}^{j-1} \sum_{c=1}^{k} \frac{s}{c}\left(\begin{array}{c}
k-1 \\
c-1
\end{array}\right)\left(\begin{array}{c}
s-k-1 \\
c-1
\end{array}\right)\left(\begin{array}{c}
s-k-c \\
j-k
\end{array}\right)
$$

Proof Consider a proper subset $W$ of $V$ with $|W|=j \geq 2$. As already observed, $\tilde{H}_{0}(\Delta(G[W]))$ will contribute (by 1$)$ to $\beta_{j-2, j}\left(I\left(C_{2 s}^{b c}\right)\right)$ in Hochster's Formula if and only if $G^{c}[W]$ is not connected.

By Remark 3.1, if $G^{c}[W]$ is not connected then $\left|W_{X}\right|>0,\left|W_{Y}\right|>0$ and $\left|N_{C_{2 s}}\left(W_{X}\right)\right|+\left|W_{Y}\right| \leq|Y|=s$. Thus, $\left|W_{Y}\right| \leq s-\left|N_{C_{2 s}}\left(W_{X}\right)\right|<s-\left|W_{X}\right|$ by Lemma 3.3.2 since $W_{X} \neq \emptyset$ and hence $\left|\operatorname{comp}\left(C_{X}\left[W_{X}\right]\right)\right| \neq 0$. This implies that $0<\left|W_{X}\right|<|W|=\left|W_{X}\right|+\left|W_{Y}\right|<s$. Thus if $|W| \geq s, G^{c}[W]$ is connected and 1 follows.

Now for $j$ with $2 \leq j \leq s-1$, we have to count how many subsets $W$ of $V$ with $|W|=j$ satisfy the condition that $G^{c}[W]$ is not connected. For each choice of $W_{X}$ with $k$ elements $\left(1 \leq k \leq j-1\right.$ in order to have $W_{X} \neq \varnothing$ and $\left.W_{Y} \neq \emptyset\right)$, we must choose $j-k$ elements from $Y \backslash N_{C_{2 s}}\left(W_{X}\right)$ for $W_{Y}$. So there are $\left(\begin{array}{c}s-\left|N_{C_{2 s}}\left(W_{X}\right)\right| \\ j-k\end{array}\right)=$ $\left(\begin{array}{c}s-k-\left|\operatorname{comp}\left(C_{X}\left[W_{X}\right]\right)\right| \\ j-k\end{array}\right)$ possible choices by Lemma 3.3.2. If we fix the number of connected components of $C_{X}\left[W_{X}\right]$ and denote it by $c$, according to [7, Lemma 3.3], there are $\frac{s}{c}\left(\begin{array}{c}k-1 \\ c-1\end{array}\right)\left(\begin{array}{c}s-k-1 \\ c-1\end{array}\right)$ possible subsets $W_{X}$ with $\left|W_{X}\right|=k$ and $\left|\operatorname{comp}\left(C_{X}\left[W_{X}\right]\right)\right|=c$, and the result follows.

Corollary 3.1 The first and the last nonzero entries on the first row of the Betti diagram of $I\left(C_{2 s}^{b c}\right)$ coincide, i.e., $\beta_{s-3, s-1}\left(I\left(C_{2 s}^{b c}\right)\right)=\beta_{0,2}\left(I\left(C_{2 s}^{b c}\right)\right)$. 
Proof For $j=s-1$ one has $\left(\begin{array}{c}s-k-c \\ j-k\end{array}\right) \neq 0$ if and only if $c=1$. In this case $\left(\begin{array}{c}k-1 \\ c-1\end{array}\right)=\left(\begin{array}{c}s-k-1 \\ c-1\end{array}\right)=\left(\begin{array}{c}s-k-c \\ j-k\end{array}\right)=1$, and hence $\beta_{s-3, s-1}\left(I\left(C_{2 s}^{b c}\right)\right)=\sum_{k=1}^{s-2} s=s(s-2)=$ $\left|E\left(C_{2 s}^{b c}\right)\right|=\beta_{0,2}\left(I\left(C_{2 s}^{b c}\right)\right)$.

The description of the Betti diagram of $I\left(C_{2 s}^{b c}\right)$ will be complete once we give its the second row. This is our next result.

\section{Proposition 3.5}

1. For all $j \geq 2 s-1, \beta_{j-3, j}\left(I\left(C_{2 s}^{b c}\right)\right)=0$.

2. For $j=4, \ldots, 2 s-2$,

$$
\beta_{j-3, j}\left(I\left(C_{2 s}^{b c}\right)\right)=\sum_{m=2}^{\lfloor j / 2\rfloor}(m-1) \sum_{a=0}^{j-2 m} \frac{2 s}{m}\left(\begin{array}{c}
j-m-a-1 \\
m-1
\end{array}\right)\left(\begin{array}{c}
2 s-j-1 \\
m-1
\end{array}\right)\left(\begin{array}{c}
2 s-j-m \\
a
\end{array}\right) .
$$

Proof By Proposition 3.2, $\tilde{H}_{1}(\Delta(G[W]))$ will contribute to $\beta_{j-3, j}\left(I\left(C_{2 s}^{b c}\right)\right)$ in Hochster's Formula (4) if and only if $W$ is a proper subset of $V$ with $|W|=j \geq 4$ such that $C_{2 s}[W]$ has at least 2 connected components that are not isolated vertices. More precisely, denoting by $w(j, m)$ the number of proper subsets $W$ of $V$ with $|W|=j$ and such that $C_{2 s}[W]$ has $m$ connected components that are not isolated vertices, then

$$
\beta_{j-3, j}(I(G))=\sum_{m=2}^{\left\lfloor\frac{j}{2}\right\rfloor}(m-1) w(j, m) .
$$

In particular, since for any subset $W$ of $V$ with $2 s-1$ elements, one sees that $C_{2 s}[W]$ is connected, 1 follows.

Now for $j \leq 2 s-2$, denote by $W(j, m, a)$ the set of proper subsets $W$ of $V$ with $|W|=j$ and such that $C_{2 s}[W]$ has $a$ isolated vertices and $m$ connected components that are not isolated vertices. Then, $w(j, m)=\sum_{a=0}^{j-2 m} w(j, m, a)$ where $w(j, m, a)=|W(j, m, a)|$, and we are reduced to compute $w(j, m, a)$ for all possible $j, m, a$.

As in the proof of [7, Lemma 3.3], observe that a subset $W$ of $V$ can be represented as a vector of length $2 s$ whose $\ell$ th entry is 1 if the $\ell$ th element in $V$ belongs to $W, 0$ otherwise. Using this correspondence, the number of nonzero entries in this vector is the number of vertices in $C_{2 s}[W]$ and the number of blocks of nonzero entries is related to the number of connected components of $C_{2 s}[W]$. In order to avoid distinguishing cases as when the vector starts/ends with $1 / 0$, we will allow to modify the starting vertex and focus only on vectors whose first entry is 1 and last entry is 0 . Denote by $B(2 s, j, k)$ the set of vectors of length $2 s$, with entries in $\{0,1\}$, whose first entry is 1 and last entry is 0 , and whose $j$ nonzero entries are located in $k$ different blocks. Let $H(j, m, a)$ be the subset of $B(2 s, j, m+a)$ formed by vectors with $m$ blocks of 1's of length strictly bigger than 1 and $a$ blocks of 1 's of length 1 and whose first block of nonzero entries has length strictly bigger than 1. Each element $w$ in $H(j, m, a)$ corresponds to $2 s$ elements in $W(j, m, a)$ (one for each choice of a vertex of $C_{2 s}$ as the vertex corresponding to the first entry of $w$ ), and an element $W$ in 
$W(j, m, a)$ corresponds to $m$ distinct elements in $H(j, m, a)$ (one for each connected component of $C_{2 s}[W]$ that we choose as the one that gives the first block of nonzero entries in the vector). Thus, $w(j, m, a)=\frac{2 s}{m}|H(j, m, a)|$.

Finally, in order to determine $|H(j, m, a)|$, note that an element in $H(j, m, a)$ comes from a vector $h$ in $B(2 s-m-a, j-m-a, m)$ by adding 1 in each block of 1 's of $h$ (there are $m$ ), and by inserting $a$ times a 1 between two zero entries of $h$. As already observed in the proof of [7, Lemma 3.3], $|B(2 s-m-a, j-m-a, m)|=$ $\left(\begin{array}{c}j-m-a-1 \\ m-1\end{array}\right)\left(\begin{array}{c}2 s-j-1 \\ m-1\end{array}\right)$. Moreover, for any element in $B(2 s-m-a, j-m-a, m)$, each block of zero entries of length $\ell$ will give $\ell-1$ places where one can add a 1 between two zero entries, and since an element in $B(2 s-m-a, j-m-a, m)$ has $2 s-j$ zero entries located in $m$ different blocks, each element in $B(2 s-m-a, j-m-a, m)$ will provide $\left(\begin{array}{c}2 s-j-m \\ a\end{array}\right)$ elements in $H(j, m, a)$. Putting all the pieces together, one gets that $w(j, m, a)=\frac{2 s}{m}\left(\begin{array}{c}2 s-j-m \\ a\end{array}\right)\left(\begin{array}{c}j-m-a-1 \\ m-1\end{array}\right)\left(\begin{array}{c}2 s-j-1 \\ m-1\end{array}\right)$ and we are done.

Corollary 3.2 The first and the last nonzero entries on the second row of the Betti diagram of $I\left(C_{2 s}^{b c}\right)$ coincide, i.e., $\beta_{2 s-5,2 s-2}\left(I\left(C_{2 s}^{b c}\right)\right)=\beta_{1,4}\left(I\left(C_{2 s}^{b c}\right)\right)$.

Proof For $j=2 s-2,\left(\begin{array}{c}2 s-j-1 \\ m-1\end{array}\right) \neq 0$ if and only if $m=2$, and then $\left(\begin{array}{c}2 s-j-m \\ a\end{array}\right) \neq 0$ if and only if $a=0$, and hence $\beta_{2 s-5,2 s-2}\left(I\left(C_{2 s}^{b c}\right)\right)=\frac{2 s}{2}\left(\begin{array}{c}2 s-5 \\ 1\end{array}\right)=s(2 s-5)$. On the other hand, $\beta_{1,4}\left(I\left(C_{2 s}^{b c}\right)\right)=\frac{2 s}{2}\left(\begin{array}{l}1 \\ 1\end{array}\right)\left(\begin{array}{c}2 s-5 \\ 1\end{array}\right)\left(\begin{array}{c}2 s-6 \\ 0\end{array}\right)$ and we are done.

Remark 3.2 Two edges $\{u, v\}$ and $\{w, z\}$ of a graph $G$ are called 3-disjoint if the induced subgraph of $G$ on $\{u, v, w, z\}$ consists of exactly two edges or, equivalently, if in the complement of $G$, the induced subgraph on $\{u, v, w, z\}$ is a 4-cycle. The maximum number of pairwise 3-disjoint edges in $G$ is called the induced matching number of $G$ and denoted by $a(G)$. By [15, Lemma 2.2], $a(G)+1 \leq \operatorname{reg}(I(G))$. In the bipartite case, it is known that equality holds for some special subclasses like unmixed bipartite graphs ([16]) or sequentially Cohen-Macaulay bipartite graphs ([19]). The family $\left\{C_{2 s}^{b c}\right\}_{s \geq 4}$ generalizes the example given in [16] of a bipartite graph whose regularity is strictly greater than $a(G)+1$. One can easily observe that $a\left(C_{6}^{b c}\right)=3$ and $a\left(C_{2 s}^{b c}\right)=2$ for all $s>3$. Hence, the induced matching number of $C_{2 s}^{b c}$ and the regularity of $I\left(C_{2 s}^{b c}\right) \subset R$ are related as follows:

$$
\operatorname{reg}\left(I\left(C_{2 s}^{b c}\right)\right)= \begin{cases}a\left(C_{2 s}^{b c}\right)+1 & \text { if } s=3 \\ a\left(C_{2 s}^{b c}\right)+2 & \text { if } s \geq 4\end{cases}
$$

This is not the only difference between the cases $s=3$ and $s \geq 4$. Indeed, $R / I\left(C_{6}^{b c}\right)$ is a complete intersection while, for $s \geq 4, R / I\left(C_{2 s}^{b c}\right)$ is not even Cohen-Macaulay (if it was then it would be Gorenstein which is impossible since its Betti diagram is not symmetric).

\section{Regularity 3 in bipartite edge ideals}

In this section we focus on edge ideals associated to bipartite graphs, which we call bipartite edge ideals. We can restrict ourselves to connected graphs since the Betti 
numbers of the edge ideal associated to a disconnected graph can be computed from the Betti numbers of the edge ideals associated to its connected components; see [14, Lemma 2.1].

Bipartite edge ideals having regularity 2 can be characterized using Theorem 2.2. They are shown to be the edge ideals associated to Ferrer's graphs in [3, Theorem 4.2].

Our aim here is to prove our main results, Theorems 4.1 and 4.2. The first one, analogous to Fröberg's classical Theorem 2.2, provides a combinatorial characterization of bipartite edge ideals having regularity 3 . The second one, analogous to Theorem 2.3, gives some extra information when the bipartite edge ideal $I(G)$ has regularity $>3$ : we determine the first step $i$ in the minimal graded free resolution of $I(G)$ where there are syzygies contributing to a graded Betti number located outside the first two rows of the Betti diagram. We also show that these syzygies are then concentrated in degree $i+4$ and compute the corresponding graded Betti number $\beta_{i, i+4}(I(G))$.

Theorem 4.1 Let $G$ be a connected bipartite graph. The edge ideal $I(G)$ has regularity 3 if and only if $G^{c}$ has at least one induced cycle (of length $\left.\geq 4\right)$ and $G^{b c}$ does not have any induced cycle of length $\geq 6$.

Theorem 4.2 Let $G$ be a connected bipartite graph and set $r:=|V(G)|$. Assume that $\operatorname{reg}(I(G))>3$ and let $t=2 s \geq 6$ be the minimal length of an induced cycle in $G^{b c}$. Then:

- $\beta_{i, j}(I(G))=0$ for all $i<t-4$ and $j>i+3$;

- $\beta_{t-4, t}(I(G))=\mid\left\{\right.$ induced $t$-cycles in $\left.G^{b c}\right\} \mid$;

- $\beta_{t-4, j}(I(G))=0$ for all $j>t$;

- for any $\boldsymbol{m} \in \mathbb{N}^{r}$ such that $|\boldsymbol{m}|=t$, one has $\beta_{t-4, \boldsymbol{m}}(I(G))=1$ if $\boldsymbol{m} \in\{0,1\}^{r}$ and $G^{b c}[W] \simeq C_{t}$ where $W:=\left\{v_{i} \in V(G): m_{i}=1\right\}$. Otherwise, $\beta_{t-4, m}(I(G))=0$.

Before we prove these results, let us recall a construction and some results from [4] that will be useful. Given a simplicial complex $\Gamma$ on the vertex set $X=\left\{x_{1}, \ldots, x_{n}\right\}$ whose facets are denoted by $F_{1}, \ldots, F_{m}$, consider $m$ new vertices, $Y:=\left\{y_{1}, \ldots, y_{m}\right\}$, and define a new simplicial complex, $\Delta(\Gamma)$, on the vertex set $X \sqcup Y$ by

$$
\Delta(\Gamma):=\Delta^{\prime} \cup \Delta_{X}
$$

where $\Delta^{\prime}:=\left\{\sigma \cup \tau: \sigma \in \Gamma, \tau \subset\left\{y_{j}: \sigma \subset F_{j}\right\}\right\}$ and $\Delta_{X}$ is the $(n-1)$-simplex on the vertex set $X$. Then, [4, Theorem 4.7] states that

$$
\tilde{H}_{i+1}(\Delta(\Gamma)) \simeq \tilde{H}_{i}(\Gamma), \quad \forall i \geq 0 .
$$

Let $G$ be a connected bipartite graph on the vertex set $V(G)=X \sqcup Y$ with $X=\left\{x_{1}, \ldots, x_{n}\right\}, Y=\left\{y_{1}, \ldots, y_{m}\right\}$, set $R:=\mathbb{K}\left[x_{1}, \ldots, x_{n}, y_{1}, \ldots, y_{m}\right]$, and denote as before $W_{X}:=W \cap X, W_{Y}:=W \cap Y$ for any subset $W$ of $V(G)$. One finds that the set

$$
\Gamma_{G}:=\left\{\sigma: \sigma \subset N_{G^{b c}}(y) \text { for some } y \in Y\right\}
$$

is a simplicial complex on $X \backslash\left\{x \in X\right.$ that are isolated vertices of $\left.G^{b c}\right\}$. 
Definition 4.1 We say that a subset $W \subset V(G)$ is relevant if $|W| \geq 3$ and $N_{G[W]}(u) \not \subset N_{G[W]}(v)$ for all $u, v \in W$ with $u \neq v$.

Lemma 4.1 If $W \subset V(G)$ is relevant, then $\Delta\left(\Gamma_{G[W]}\right)=\Delta(G)[W]$.

Proof Denote by $\Gamma:=\Gamma_{G[W]}$ the simplicial complex associated to the graph $G[W]$ as in (8), let $\mathcal{F}(\Gamma)$ be its set of facets, and set $\Delta:=\Delta(\Gamma)$ as defined in (6). Since $W$ is relevant, $G[W]$ has no isolated vertex and hence $W_{X}$ is the vertex set of $\Gamma$. Moreover, $\mathcal{F}(\Gamma)=\left\{N_{G^{b c}[W]}(y): y \in W_{Y}\right\}$. This implies that $\Delta=\Delta^{\prime} \cup \Delta_{W_{X}}$ where $\Delta^{\prime}=\left\{\sigma \cup \tau: \sigma \in \Gamma_{G[W]}, \tau \subset\left\{y \in W_{Y}: \sigma \subset N_{G^{b c}[W]}(y)\right\}\right\}$. Consider $\sigma \subset W$. If $\sigma \subset$ $W_{X}$ then $\sigma \in \Delta_{W_{X}} \subset \Delta$ and also $\sigma \in \Delta(G)[W]$. Otherwise, one has

$$
\begin{aligned}
\sigma \in \Delta \backslash \Delta_{W_{X}} & \Leftrightarrow \sigma_{X} \in \Gamma_{G[W]}, \sigma_{Y} \neq \emptyset \text { and } \sigma_{X} \subset N_{G^{b c}[W]}(y), \quad \forall y \in \sigma_{Y} \\
& \Leftrightarrow \sigma_{Y} \neq \emptyset \text { and } \sigma_{X} \subset N_{G^{b c}[W]}(y), \quad \forall y \in \sigma_{Y} \\
& \Leftrightarrow \sigma_{Y} \neq \emptyset \text { and }\{x, y\} \notin E(G[W]), \quad \forall x \in \sigma_{X}, \forall y \in \sigma_{Y} \\
& \Leftrightarrow \sigma \not \subset W_{X} \text { and } \sigma \in \Delta(G)[W] \\
& \Leftrightarrow \sigma \in \Delta(G)[W] \backslash \Delta_{W_{X}} .
\end{aligned}
$$

Thus, $\sigma \in \Delta \Leftrightarrow \sigma \in \Delta(G)[W]$.

Lemma 4.2 Let $I:=I_{\Gamma_{G[W]}}$ be the Stanley-Reisner ideal associated to $\Gamma_{G[W]}$ and let $\left\{m_{1}, \ldots, m_{s}\right\}$ be its monomial minimal generating set. One has:

- if $W$ is relevant then $\operatorname{deg}\left(m_{i}\right) \geq 2, \forall i \in[s]$;

- $\max \left\{\operatorname{deg}\left(m_{i}\right): i \in[s]\right\} \leq a(G[W])$.

Proof If $W$ is relevant and $x \in W$ then $N_{G[W]}(x) \subsetneq W_{Y}$. Thus, $x \in N_{G^{b c}[W]}(y)$ for some $y \in W_{Y}$, and hence $\{x\} \in \Gamma_{G[W]}$. Therefore, non-faces must have dimension strictly greater than 1 . Since minimal generators of $I$ correspond to minimal nonfaces of $\Gamma_{G[W]}$, the first claim follows.

If $g=x_{i_{1}} \cdots x_{i_{d}}$ is a minimal generator of $I,\left\{x_{i_{1}}, \ldots, x_{i_{d}}\right\} \not \subset N_{G^{b c}[W]}(y)$ for all $y \in W_{Y}$ and $\frac{g}{x_{i_{k}}} \notin I$ for all $k \in[d]$. Hence, for every $l \in[d], F_{l}:=\left\{x_{i_{k}}\right.$ : $k \neq l\} \subset N_{G^{b c}[W]}(y)$ for some element $y$ in $W_{Y}$ that we denote by $y(l)$. Then, $x_{i_{l}} \notin N_{G^{b c}[W]}(y(l))$, or equivalently, $x_{i_{l}} \in N_{G[W]}(y(l))$ and $x_{i_{k}} \notin N_{G[W]}(y(l))$ if $k \neq l$. So $\left\{\left\{x_{i_{l}}, y(l)\right\}: l \in[d]\right\}$ is a set consisting of $d$ disconnected edges of $G[W]$. This implies that $a(G[W]) \geq d$.

Proof (of Theorems 4.1 and 4.2) We first prove the equivalence in Theorem 4.1 and show that the extra information contained in Theorem 4.2 then follows quite easily. First assume that $\operatorname{reg}(I(G))=3$. By Theorem 2.2, $G^{c}$ contains an induced cycle of length $l \geq 4$. Moreover, if there exists a subset $W$ of $V(G)$ such that $G^{b c}[W] \simeq C_{l}$ for some (even) $l \geq 6$ then, since on the one hand $\beta_{i, j}(I(G)) \geq \beta_{i, j}(I(G[W]))$ for all $i, j$ by Hochster's Formula (4), and on the other $\beta_{l-4, l}\left(I\left(C_{l}^{b c}\right)\right)=1$ by Theorem 3.1, one gets that $\operatorname{reg}(I(G)) \geq 4$, a contradiction. 
Conversely, assume that $\operatorname{reg}(I(G)) \neq 3$. If $\operatorname{reg}(I(G))=2$ then there is no induced cycle in $G^{c}$ by Theorem 2.2 and the result holds. If $\operatorname{reg}(I(G))>3$ then, by Theorem 2.1, there exists $i$ such that $\beta_{i, i+4}(I) \neq 0$. Denote by $i_{4}$ the smallest integer with this property. By [15, Lemma 2.2], $i_{4} \geq 2$ and if $i_{4}=2$, then $\beta_{2,6}(I) \neq 0$ is the number of induced subgraphs of $G$ isomorphic to $3 K_{2}$. We only have to notice that $\left(3 K_{2}\right)^{b c} \simeq C_{6}$ to obtain the result that if $i_{4}=2, G^{b c}$ contains an induced cycle of length 6. On the other hand, all the items in Theorem 4.2 follow in this case from Theorem 2.1 and [9, Theorem 2.1] which states that, for any monomial in $R, \boldsymbol{x}^{\boldsymbol{m}}$, if one collects at each step of the minimal multigraded free resolution of $I(G)$, the minimal generators whose multidegree divides $\boldsymbol{x}^{\boldsymbol{m}}$, one gets a minimal multigraded free resolution of $I(G)_{m}$, the edge ideal whose minimal generators divide $\boldsymbol{x}^{\boldsymbol{m}}$.

If $i_{4} \geq 3$, by (4) there exists $W \subset V(G)$ such that

$$
|W|=i_{4}+4 \text { and } \operatorname{dim}_{\mathbb{K}}\left(\tilde{H}_{2}(\Delta(G)[W])\right)>0 .
$$

As in the case $i_{4}=2$, we will be done using Theorem 2.1 and [9, Theorem 2.1] if we show that the subsets $W \subset V(G)$ satisfying (9) are the ones such that

$$
G[W] \simeq\left(C_{i_{4}+4}\right)^{b c} .
$$

If $W$ satisfies (10), then it satisfies (9) by Proposition 3.1. Now take $W$ satisfying (9). If $W$ is not relevant, there exist $u, v \in W$ such that $N_{G[W]}(u) \subset N_{G[W]}(v)$ and $\tilde{H}_{i}(\Delta(G[W])) \simeq \tilde{H}_{i}(\Delta(G[W \backslash\{v\}])), \forall i \geq 0$ by Lemma 2.1.5. The proper subset $W \backslash\{v\}$ of $W$ then contradicts the definition of $i_{4}$ so $W$ has to be relevant. Set

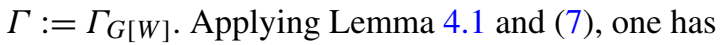

$$
\operatorname{dim}_{\mathbb{K}}\left(\tilde{H}_{1}(\Gamma)\right)=\operatorname{dim}_{\mathbb{K}}\left(\tilde{H}_{2}(\Delta(G)[W])\right)>0 .
$$

Moreover, $\operatorname{dim}_{\mathbb{K}}\left(\tilde{H}_{1}\left(\Gamma\left[X^{\prime}\right]\right)\right)=0$ for all $X^{\prime} \subsetneq W_{X}$ since $\Delta\left(\Gamma_{X^{\prime}}\right) \simeq \Delta(G)\left[W^{\prime}\right]$ where $W^{\prime}=X^{\prime} \sqcup W_{Y}$ and if $\operatorname{dim}_{\mathbb{K}}\left(\tilde{H}_{1}\left(\Gamma\left[X^{\prime}\right]\right)\right)=\operatorname{dim}_{\mathbb{K}}\left(\tilde{H}_{2}\left(\Delta(G)\left[W^{\prime}\right]\right)\right)>0$, we will reach a contradiction with the minimality of the size of $W$.

As $i_{4}>2$, we have $\beta_{2,6}(I(G))=0$ and hence, by [15, Lemma 2.2], $a(G)=2$. Thus, by Lemma 4.2, $I_{\Gamma}$ is generated in degree 2, i.e., it is an edge ideal, and hence we can write $\Gamma=\Delta\left(G^{*}\right)$ for some simple graph $G^{*}$ on the vertex set $W_{X}$. Thus, $\operatorname{dim}_{\mathbb{K}}\left(\tilde{H}_{1}\left(\Delta\left(G^{*}\right)\right)\right)>0$ and $\operatorname{dim}_{\mathbb{K}}\left(\tilde{H}_{1}\left(\Delta\left(G^{*}\right)\left[X^{\prime}\right]\right)\right)=0$ for all $X^{\prime} \subsetneq W_{X}$. Applying Theorem 2.3, we have $C_{l}<\left(G^{*}\right)^{c}$ for some $l \geq 4$ but $C_{l} \nless\left(G^{*}\right)^{c}\left[X^{\prime}\right]$ for all $X^{\prime} \subsetneq W_{X}$, so necessarily, $\left(G^{*}\right)^{c}=C_{l}$ and $l=\left|W_{X}\right|$. Therefore, $\Gamma=\Delta\left(C_{l}^{c}\right)=C_{l}$ and we have $\left|N_{G^{b c}[W]}(y)\right|=2$, for all $y \in W_{Y}$. Together with the fact that $N_{G^{b c}[W]}(u) \not \subset$ $N_{G^{b c}[W]}(v)$ for all $u, v \in W$ such that $u \neq v$ (so $\left|N_{G^{b c}[W]}(u)\right| \neq 1, u \in W_{X}$ ) and that $\sum_{u \in W_{X}} \operatorname{deg}_{G^{b c}[W]}(u)=\sum_{v \in W_{Y}} \operatorname{deg}_{G^{b c}[W]}(v)$ (so $\left|N_{G^{b c}[W]}(u)\right| \leq 2, u \in W_{X}$ ), this implies that $\left|N_{G^{b c}[W]}(y)\right|=2$ for all $y \in W$. Moreover, $G^{b c}[W]$ is connected because $\Gamma$ is, and hence $G^{b c}[W] \simeq C_{|W|}$.

Remark 4.1 One can find in [15] several examples of edge ideals whose regularity is 3 or 4 depending on the characteristic of the field $\mathbb{K}$. This shows that in Theorem 4.1 the bipartite hypothesis cannot be removed. 
The following example constructed in [4] shows that it is hopeless to give a combinatorial characterization of bipartite edge ideals of regularity 4 .

Example 4.1 (see [4, Example 4.8]) Consider the following bipartite edge ideal:

$$
\begin{aligned}
I(G)= & \left(x_{1} y_{1}, x_{2} y_{1}, x_{3} y_{1}, x_{7} y_{1}, x_{9} y_{1}, x_{1} y_{2}, x_{2} y_{2}, x_{4} y_{2}, x_{6} y_{2}, x_{10} y_{2},\right. \\
& x_{1} y_{3}, x_{3} y_{3}, x_{5} y_{3}, x_{6} y_{3}, x_{8} y_{3}, x_{2} y_{4}, x_{4} y_{4}, x_{5} y_{4}, x_{7} y_{4}, x_{8} y_{4}, \\
& \left.x_{3} y_{5}, x_{4} y_{5}, x_{5} y_{5}, x_{9} y_{5}, x_{10} y_{5}, x_{6} y_{6}, x_{7} y_{6}, x_{8} y_{6}, x_{9} y_{6}, x_{10} y_{6}\right) .
\end{aligned}
$$

Then, $\operatorname{reg}(I(G))=4$ if $\operatorname{char}(\mathbb{K})=0$ and $\operatorname{reg}(I(G))=5$ if $\operatorname{char}(\mathbb{K})=2$.

\section{The non-square-free case}

Let $I$ be an ideal in $R:=\mathbb{K}\left[x_{1}, \ldots, x_{n}\right]$ generated by monomials of degree two which is not square-free. Assume, without loss of generality, that $I$ is minimally generated by $\left\{m_{1}, \ldots, m_{s}\right\}$ where $m_{1}=x_{1}^{2}, \ldots, m_{l}=x_{l}^{2}$ and $m_{l+1}, \ldots, m_{s}$ are square-free for some $l \in[s]$. We define

- $I_{\text {sqf }}:=\left(m_{l+1}, \ldots, m_{s}\right) \subset R$, and

- $I_{\mathrm{pol}}:=\left(x_{1} y_{1}, \ldots, x_{l} y_{l}, m_{l+1}, \ldots, m_{s}\right) \subset R^{*}:=\mathbb{K}\left[x_{1}, \ldots, x_{n}, y_{1}, \ldots, y_{l}\right]$.

The ideal $I_{\mathrm{pol}}$, called the polarization of $I$, has the following useful property ([10, Corollary 1.6.3]): if we provide $R$ and $R^{*}$ with a $\mathbb{N}^{n}$-multigrading such that $\operatorname{deg}\left(x_{i}\right)=\boldsymbol{e}_{i}$ for all $i \in[n]$ and $\operatorname{deg}\left(y_{j}\right)=\boldsymbol{e}_{j}$ for all $j \in[l]$, then

$$
\beta_{i, \boldsymbol{m}}(I)=\beta_{i, \boldsymbol{m}}\left(I_{\mathrm{pol}}\right), \quad \forall i \geq 0, \forall \boldsymbol{m} \in \mathbb{N}^{n} .
$$

Both ideals $I_{\mathrm{sqf}}$ and $I_{\mathrm{pol}}$ are edge ideals. We will call $G$ the non-simple graph associated to $I$ and denote, as in the square-free case, $I=I(G)$. Denote by $G_{\text {sqf }}$ and $G_{\mathrm{pol}}$ the simple graphs associated to $I_{\mathrm{sqf}}$ and $I_{\mathrm{pol}}$, respectively.

Definition 5.1 We say that two edges $e_{1}, e_{2} \in E(G)$ are totally disjoint provided $\{u, v\} \notin E(G)$ if $u \in e_{1}$ and $v \in e_{2}$.

Assume that the simple graph $G_{\text {sqf }}$ is connected and bipartite. We say that the non-simple graph $G$ is bipartite and define the bipartite complement of $G$ as the bipartite complement of the simple graph $G_{\mathrm{sqf}}$, i.e., $G^{b c}:=\left(G_{\mathrm{sqf}}\right)^{b c}$. We also define the complement of $G$ as $G^{c}:=\left(G_{\mathrm{sqf}}\right)^{c}$.

We can complete the characterization of ideals associated to bipartite graphs having regularity 3 with the non-square-free case as follows:

Proposition 5.1 Let $I \subset R$ be a non-square-free monomial ideal generated in degree two and assume that the non-simple graph $G$ associated to $I$ is bipartite. Then, I has regularity 3 if and only if

- $G$ either has two totally disjoint edges or $C_{l}<G^{c}$ for some $l \geq 5$, 
- $G$ does not have three edges that are pairwise totally disjoint, and

- $G^{b c}$ has no induced cycle of length $\geq 8$.

Proof By (11), $\operatorname{reg}(I)=3$ if and only if $\operatorname{reg}\left(I_{\mathrm{pol}}\right)=3$ and, using Theorem 4.1 , this occurs if and only if $\left(G_{\mathrm{pol}}\right)^{c}$ has an induced cycle of length 4 and $\left(G_{\mathrm{pol}}\right)^{b c}$ has no induced cycle of length $\geq 6$. Rewriting these properties of the graph $G_{\text {pol }}$ in terms of the graph $G$, the result follows.

When $\operatorname{reg}(I)>3$, the claims in Theorem 4.2 remain valid if $G$ does not contain three edges that are pairwise totally disjoint since $l$-cycles in $\left(G_{\mathrm{sqf}}\right)^{c}$ and in $\left(G_{\mathrm{sqf}}\right)^{b c}$ coincide with the $l$-cycles in $\left(G_{\mathrm{pol}}\right)^{c}$ and $\left(G_{\mathrm{pol}}\right)^{b c}$, respectively, provided $l>6$. However, if $G$ has three edges that are pairwise totally disjoint, then:

- $\beta_{i, j}(I)=0$ if $i \leq 1$ and $j>i+3$;

- $\beta_{2,6}(I)$ is the number of induced subgraphs of $G$ isomorphic to three pairwise totally disjoint edges;

- $\beta_{2, j}(I)=0$ for all $j>6$;

- considering the $\mathbb{N}^{n}$-multigrading on $R$, for all $\boldsymbol{m} \in \mathbb{N}^{n}$ such that $|\boldsymbol{m}|=6$, one has $\beta_{2, m}(I)=1$ if $G\left[\left\{x_{i}: m_{i}=1\right\}\right]$ consists of three totally disjoint edges. Otherwise, $\beta_{2, \boldsymbol{m}}(I)=0$.

Example 5.1 The ideal $I=\left(x_{1}^{2}, x_{1} x_{5}, x_{2} x_{5}, x_{2} x_{7}, x_{3} x_{5}, x_{3} x_{6}, x_{3} x_{7}, x_{4} x_{6}\right)$ satisfies the condition $\beta_{2,6}(I)=1$. The bipartite graph $G^{b c}$ does not have any induced 6-cycle but there are three pairwise disjoint edges in $G$.

Acknowledgements Both authors were partially supported by Ministerio de Ciencia e Innovación (Spain), MTM2010-20279-C02-02. The first author is supported by an INdAM-COFUND Marie Curie Fellowship (Italy).

\section{References}

1. Avramov, L., Conca, A., Iyengar, S.: Free resolutions over commutative Koszul algebras. Math. Res. Lett. 17, 197-210 (2010)

2. Björner, A.: Topological methods. In: Handbook of Combinatorics, vol. 1, pp. 1819-1872. Elsevier, Amsterdam (1995)

3. Corso, A., Nagel, U.: Monomial and toric ideals associated to Ferrers graphs. Trans. Am. Math. Soc. 361, 1371-1395 (2009)

4. Dalili, K., Kummini, M.: Dependence of Betti numbers on characteristic. Commun. Algebra (2010, to appear). arXiv:1009.4243

5. Diestel, R.: Graph Theory, 4th edn. Graduate Texts in Mathematics, vol. 173. Springer, Heidelberg (2010)

6. Eisenbud, D., Green, M., Hulek, K., Popescu, S.: Restricting linear syzygies: algebra and geometry. Compos. Math. 141, 1460-1478 (2005)

7. Fernández-Ramos, O., Gimenez, P.: First nonlinear syzygies of ideals associated to graphs. Commun. Algebra 37, 1921-1933 (2009)

8. Fröberg, R.: On Stanley-Reisner rings. In: Topics in Algebra, Part 2 (Warsaw, 1988), vol. 26, pp. 57-70. Banach Center, Warsaw (1990)

9. Gasharov, V., Hibi, T., Peeva, I.: Resolutions of $a$-stable ideals. J. Algebra 254, 375-394 (2002)

10. Herzog, J., Hibi, T.: Monomial Ideals. Graduate Texts in Mathematics, vol. 260. Springer, London (2011) 
11. Herzog, J., Srinivasan, H.: A note on the subadditivity problem for maximal shifts in free resolutions (2013). arXiv: 1303.6214

12. Hochster, M.: Cohen-Macaulay rings, combinatorics, and simplicial complexes. In: Ring Theory, II, Proc. Second Conf., Univ. Oklahoma, Norman, Okla., 1975. Lecture Notes in Pure and Appl. Math., vol. 26, pp. 171-223. Dekker, New York (1977)

13. Hà, H.T., Van Tuyl, A.: Resolutions of square-free monomial ideals via facet ideals: a survey. In: Algebra, Geometry and Their Interactions. Contemp. Math., vol. 448, pp. 91-117. Amer. Math. Soc., Providence (2007)

14. Jacques, S., Katzman, M.: The Betti numbers of forests (2005). arXiv:math.AC/0501226

15. Katzman, M.: Characteristic-independence of Betti numbers of graph ideals. J. Comb. Theory, Ser. A 113, 435-454 (2006)

16. Kummini, M.: Regularity, depth and arithmetic rank of bipartite edge ideals. J. Algebr. Comb. 30, 429-445 (2009)

17. Morey, S., Villarreal, R.H.: Edge Ideals: Algebraic and Combinatorial Properties. Progress in Commutative Algebra, vol. 1, pp. 85-126. de Gruyter, Berlin (2012)

18. Munkres, J.R.: Elements of Algebraic Topology. Addison-Wesley, Menlo Park (1984)

19. Van Tuyl, A.: Sequentially Cohen-Macaulay bipartite graphs: vertex decomposability and regularity. Arch. Math. (Basel) 93, 451-459 (2009)

20. Villarreal, R.H.: Cohen-Macaulay graphs. Manuscr. Math. 66, 277-293 (1990)

21. Villarreal, R.H.: Monomial Algebras. Monographs and Textbooks in Pure and Applied Mathematics, vol. 238. Dekker, New York (2001) 